\title{
LEISHMANIA SIAMENSIS ALS ERREGER VON AUTOCHTHONER KUTANER LEISHMANIOSE BEI PFERDEN IN DEUTSCHLAND - EINE NEUE INFEKTIONSKRANKHEIT IN MITTELEUROPA?
}

\author{
Denise Sinning, Kernt Köhler, Lutz-Ferdinand Litzke, Gabriele Schönian, Marcus Frohme, Katrin Kuhls
}

\section{Zusammenfassung}

Aus mitteleuropäischer Sicht ist die durch Parasiten verursachte und von Sandmücken übertragene Leishmaniose eine in Ländern tropischer und subtropischer Regionen auftretende Infektionskrankheit. In zunehmendem Maße werden jedoch autochthone Fälle in Mitteleuropa, insbesondere in Süddeutschland, verzeichnet. Dies ist vermutlich auf die globale Erwärmung und die Ausdehnung des Verbreitungsgebietes der Sandmücken nach Norden zurückzuführen. Die vorliegende Arbeit befasst sich mit der Identifizierung und phylogenetischen Charakterisierung der Erreger dieser Fälle. Dazu wurden verschiedene Marker im Leishmaniengenom sequenziert und mit bekannten Arten verglichen. Die untersuchte DNA stammte von autochthonen kutanen Leishmaniosen bei Pferden und einem Rind, die in den letzten zehn Jahren in Deutschland und der Schweiz auftraten. Aufgrund identischer Sequenzen konnten die Parasiten als $L$. siamensis identifiziert bzw. verifiziert werden, eine erst im Jahr 2008 neu beschriebene Art, die in Thailand humane viszerale Leishmaniose verursacht. Die phylogenetischen Analysen zeigten die Ähnlichkeit von L. siamensis mit weiteren bisher nicht identifizierten Stämmen aus Martinique und Ghana, die kutane Leishmaniose bei Menschen verursachen. Um die Frage zu beantworten, ob sich die Leishmaniose zu einer in Mitteleuropa endemischen zoonotischen Krankheit entwickeln könnte, müssen weitere Studien über kompatible Vektoren, mögliche Reservoire und zur Virulenz durchgeführt werden.

\section{Abstract}

From our Central European point of view leishmaniasis is a vector-borne parasitic disease that occurs mainly in tropical and subtropical countries. In recent years there is however an increasing number of reports of autochthonous cases in Central Europe, especially in southern Germany. This is probably caused by climate change and global warming and the northward shift of the occurrence of the sandfly vectors. The aim of the present study was the identification and phylogenetic characterization of the causative agents of these cases. To this end we sequenced several markers of the Leishmania genome and compared them with those of the known Leishmania species. The studied DNA originated from several autochthonous cases of cutaneous leishmaniasis in horses and a cow that occurred during the last decade in Germany and Switzerland. The parasites were identified or verified as $L$. siamensis, a newly described species that causes human visceral leishmaniasis in Thailand. The phylogenetic analyses also showed the similarity with previously not identified strains that caused human cutanous leishmaniasis in Ghana and Martinique. To answer the question whether leishmaniasis is becoming endemic as a zoonotic disease in Central Europe further research has to be done on vector compatibility, virulence and possible reservoire animals.

\section{EINFÜHRUNG}

Die Leishmaniose ist eine durch Vektoren übertragene Infektionskrankheit, die von obligaten Protozoen der Gattung Leishmania verursacht wird. Laut WHO [WHO 2010] ist die Leishmaniose in 88 Ländern endemisch, mit ca. 12 Mio. infizierten Menschen weltweit und jährlich ca. 2 Mio. Neuinfektionen. Das Verbreitungsgebiet der Leishmaniose umfasst die tropischen und subtropischen Regionen aller Kontinente, einschließlich des europäischen Mittelmeerraumes. Die Parasiten werden durch Phlebotomen (Sandmü- cken) übertragen. Je nach Erreger und Verbreitungsgebiet kann die Leishmaniose anthroponotisch oder zoonotisch sein, der Transmissionszyklus umfasst je nach Ökosystem spezifische Parasiten-, Sandmücken- und tierische Wirtsarten bzw. den Menschen.

Es gibt verschiedene klinische Ausprägungen der Erkrankung, bei denen entweder die Haut bzw. Schleimhaut (kutane und mukokutane Leishmaniose - CL und MCL) oder das gesamte Immunsystem und innere Organe des Infizierten (viszerale Leishmaniose, auch Kala-Azar genannt - VL) betrof- fen sind (Abb. 1), wobei die viszerale Leishmaniose bei Nichtbehandlung gewöhnlich letal verläuft. Es existieren ungefähr 30 Leishmanienarten, von denen etwa 20 im Menschen Leishmaniose auslösen können. Zu den wichtigsten gehören $L$. infantum und L. donovani (Auslöser der VL), L. major, L. tropica (Auslöser der $C L$ ) und $L$. braziliensis und L. guyanensis (Auslöser der $C L$ und $M C L$ ). Die im europäischen Mittelmeerraum auftretende Leishmaniose wird durch L. infantum verursacht.

In Deutschland tritt die Leishmaniose üblicherweise nur in Verbindung mit 

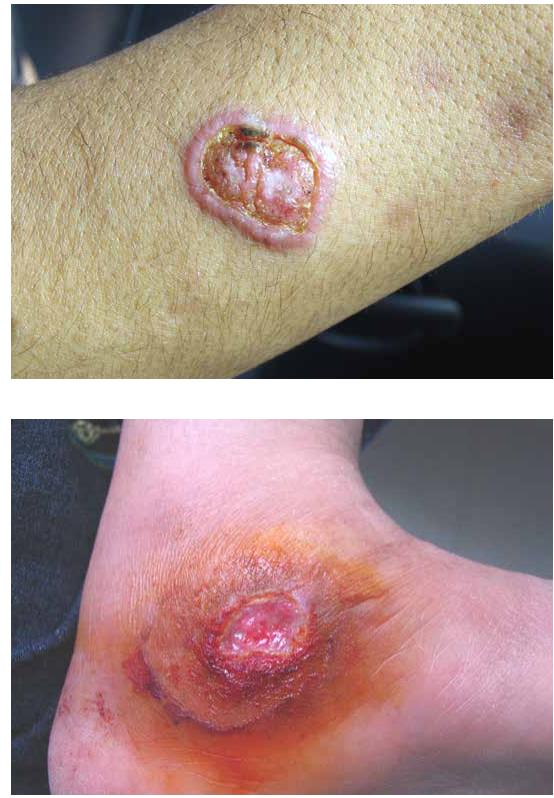

Abb. 1) Beispiele für die kutane Leishmaniose be verschiedenen Patienten (mit freundlicher Genehmigung von Dr. Amer Al-Jawabreh, Leishmaniases Research Unit, Jericho, Palestine).

Reisen in Endemiegebiete auf [Harms et al. 2003, Weitzel et al. 2005] und ist bisher nicht meldepflichtig. Im Jahr 1991 wurde jedoch der erste autochthone ( einheimische) Fall beschrieben [Gothe 1991], ca. 17 weitere kamen in den folgenden Jahren bis 2012 dazu. Zwischen 1991 und 2008 wurden 11 autochthone Fälle bestätigt, von denen jedoch nur vier publiziert wurden [Naucke et al. 2008], weitere 7 Fälle kamen bis 2012 dazu [Müller et al. 2009, Kuhls et al. 2013]. Generell sind verschiedene autochthone Fälle aus Mitteleuropa (Schweiz, Österreich, Deutschland, Niederlande) bei Menschen, Hunden und Pferden seit den 60er Jahren bekannt geworden, jedoch nur wenige davon wurden veröffentlicht (Tabelle 1). Diesen Meldungen wurde bis 1999 wenig Bedeutung zugeschrieben, da man davon ausging, dass der Vektor in diesen Ländern nicht vorkommt.

Im Jahr 1999 wurden das erste Mal Sandmücken in Deutschland (BadenWürttemberg) nachgewiesen [Naucke \& Pesson 2000, Naucke 2002], es handelte sich um Phlebotomus mascittii, dessen Transmissionspotenzial für Leishmania jedoch bisher nicht getestet wurde. Bis 2007 wurde Ph. mascittii an 16 verschiedenen Stellen in Baden-Württemberg und einer Stelle in Rheinland-Pfalz (Cochem-Mosel) gefunden. Cochem ist die bisher nördlichste Fundstelle von Sandmücken in Europa $\left(50^{\circ} 19^{\prime} 41.2^{\prime \prime N}\right)$. Im Jahr 2001 wurde Phlebotomus perniciosus (Vektor für L. infantum) das erste Mal in Deutschland entdeckt [Naucke \& Schmitt 2004]. Es wird angenommen, dass Ph. perniciosus von Frankreich über das Saarland nach Deutschland gekommen ist. Die Ausbreitung von Ph. mascittii verläuft entlang des Rheingrabens und der Mosel. Desweiteren muss man davon ausgehen, dass die bisherigen Funde nicht das eigentliche Ausmaß der Verbreitung und die Häufigkeit dieser Sandmücken widerspiegeln. Auch in Österreich (Kärnten) [Naucke et al. 2011], der Süd- und Westschweiz [Galli-Valerio 1912, Vogel 1931, Knechtli \& Jenni 1990, Grimm et al. 1990, 1993], Belgien [Depaquit et al. 2005] und Frankreich nahe der Grenze zu Deutschland [Callot 1950] treten Sandmücken ( $P h$. mascittii) auf. Die theoretische Verbreitungsgrenze stellt die $10^{\circ} \mathrm{C}$-Jahresisotherme dar. Auch das Verbreitungsgebiet von Ph. perfiliewi, eines in Norditalien zirkulierenden Vektors für L. infantum, verschiebt sich aufgrund der globalen Erwärmung derzeit nordwärts [Maroli et al. 2008], so dass diese Art in den nächsten Jahren bis zum 49. Breitengrad vorkommen wird (Baden-Württemberg, Bayern, Schweiz, Österreich). Erste Modelling-Ansätze hinsichtlich der zukünftigen Klimaveränderungen in Mitteleuropa und den Auswirkungen auf die Verbreitung von Sandmücken deuten darauf hin, dass sich die Habitat-Bedingungen bis zur Hälfte des 21. Jahrhunderts so stark ändern werden, dass sie für alle der untersuchten L. infantum Vektoren geeignet sind [Fischer et al. 2010, 2011]. Alle bisherigen autochthonen Leishmaniosen in Deutschland traten im Verbreitungsgebiet von Ph. mascittii und $\mathrm{Ph}$. perniciosus auf.

Zu den nachgewiesenen Reservoirtieren von Leishmanien ( $L$. infantum) in Europa zählen in erster Linie die Hunde, aber auch Katzen, Füchse und Nagetiere. Infektionen wurden auch bei Pferden, Eseln und Kühen gefunden. In vielen Regionen Südeuropas (z.B. auf Mallorca oder Sizilien) liegt die Infektionsrate bei Hunden bei bis zu 70 $\%$ und die Anzahl der von dort nach
Deutschland importierten Hunde nimmt zu. Man nimmt an, dass es in Deutschland ca. 20.000 infizierte Hunde - entweder durch Urlaubsreisen in die Endemiegebiete oder das Mitbringen von infizierten Hunden - gibt [Nauke et al. 2008, Menn et al. 2010]. Über diese Tiere und lokale Sandmücken in Deutschland könnten sich die Infektionen somit weiter ausbreiten.

Nur wenige der autochthonen Infektionen wurden publiziert und die Erreger auf Artebene diagnostiziert. Bis 2009 wurde davon ausgegangen, dass nur L. infantum als Erreger dieser autochthonen Fälle in Frage kommt. Umso bemerkenswerter ist es, dass bereits 2004 in Deutschland ein Fall von Hautleishmaniose bei einem Pferd auftrat, bei dem jedoch zum damaligen Zeitpunkt der Erreger keiner bis dahin bekannten Leishmaniaart zugeordnet werden konnte. Es handelte sich um einen ca. 4 Jahre alten Warmblut-Wallach mit einer Hautläsion am Augenlid (Abb. 2).

Das Pferd wurde im Bayrischen Wald geboren und kam mit ca. 6 Monaten nach Aschaffenburg. Sechs Monate

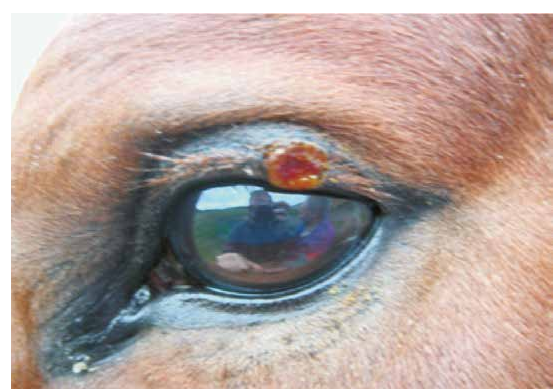

Abb. 2a) Ca. kirschgroße Umfangsvermehrung unmittelbar am Lidrand bei einem 4-jährigen Wallach (präoperativer Befund).

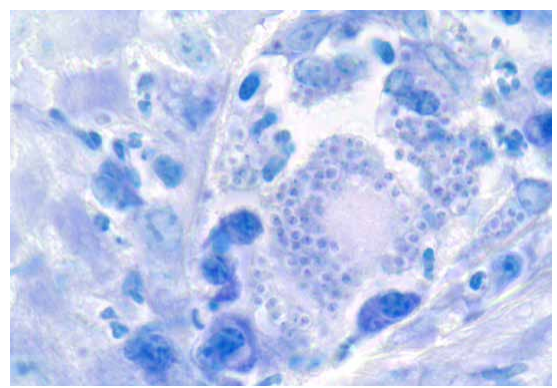

Abb. 2b) Giemsa gefärbtes Präparat mit 1000-facher Vergrößerung einer intraepithelialen Pustel mit zahlreichen Makrophagen und darin massenhaft amastigote Protozoenstadien (Leishmanien).

vor der Erkrankung wurde das Pferd nach Gießen umgestellt. Nach der chirurgischen Entfernung der Läsion wur- 


\begin{tabular}{|c|c|c|c|c|c|}
\hline Land & Region & $\begin{array}{l}\text { Jahr des Auftretens } \\
\text { der Infektion }\end{array}$ & Referenz & $\begin{array}{l}\text { Wirt (Anzahl } \\
\text { der Fälle) }\end{array}$ & Spezies \\
\hline \multirow{8}{*}{$\begin{array}{l}\text { Deutschland } \\
\text { (seit } 1991 \\
12 \text { bestätigte } \\
\text { autochthone } \\
\text { Fälle bei } \\
\text { Mensch, Hund, } \\
\text { Pferd, Katze) }\end{array}$} & $\begin{array}{l}\text { Nordrhein-Westfalen } \\
\qquad(\text { Köln) }\end{array}$ & & & Hund (1) & L. spec. \\
\hline & $\begin{array}{c}\text { Bayern } \\
\text { (Landsberg/Lech) }\end{array}$ & - & Gothe (1991) & Hund (1) & L. spec. \\
\hline & $\begin{array}{l}\text { Nordrhein-Westfalen } \\
\text { (Aachen) }\end{array}$ & 1997 & Bogdan et al. (2001) & Mensch (1) & L. infantum \\
\hline & $\begin{array}{c}\text { Bayern } \\
\text { (Augsburg) }\end{array}$ & 2000 & Koehler et al. (2002) & Pferd (1) & L. infantum \\
\hline & $\begin{array}{l}\text { Rheinland-Pfalz } \\
\text { (Gehrweiler) }\end{array}$ & 1998/99 & nicht publiziert & Hund (1) & L. spec. \\
\hline & Bayern & - & Müller et al. (2009) & Pferd (1) & L. siamensis* \\
\hline & Rheinland-Pfalz & - & Müller et al. (2009) & Pferd (5) & L. siamensis* \\
\hline & $\begin{array}{c}\text { Bayern } \\
\text { (Aschaffenburg) } \\
\text { und } \\
\text { Hessen } \\
\text { (Gießen) }\end{array}$ & 2004 & $\begin{array}{l}\text { Kuhls et al. (2013), } \\
\text { Litzke et al. (2006) }\end{array}$ & Pferd (1) & L. siamensis* \\
\hline \multirow{4}{*}{ Schweiz } & Südschweiz & - & Mazzi (1976) & Mensch & L. infantum \\
\hline & - & - & Schawalder (1977) & Hund & L. spec. \\
\hline & Nordschweiz & - & Müller et al. (2009) & Pferd (2) & L. siamensis \\
\hline & Züricher Oberland & 2009 & Lobsiger et al. (2010) & Kuh (1) & L. siamensis* \\
\hline \multirow{3}{*}{ Österreich } & Niederösterreich & - & Beyreder (1962) & Mensch & L. spec. \\
\hline & - & - & Kollaritsch et al. (1989) & Mensch & L. spec. \\
\hline & - & - & Dornbusch et al. (1999) & Mensch (1) & L. spec. \\
\hline \multirow[b]{2}{*}{ Niederlande } & - & - & Slappendel (1988) & Hund & L. spec. \\
\hline & - & - & $\begin{array}{l}\text { Diaz-Espineira \& } \\
\text { Slappendel (1997) }\end{array}$ & Hund & L. spec. \\
\hline
\end{tabular}

*Die in dieser Arbeit in die Sequenzierung einbezogenen Fälle. k. A. - keine Angaben

Tab. 1) Autochthone Leishmaniose-Fälle in Mitteleuropa

de aus dem Gewebe die Parasiten-DNA extrahiert und eine Sequenzanalyse der ITS1-Region der ribosomalen DNA sowie des DNA-Polymerase- $\alpha$-Gens durchgeführt, eine Artidentifizierung war jedoch nicht möglich [Litzke et al. 2006, Kuhls et al. 2013]. Das Pferd erlitt vier Wochen nach dem chirurgischen Eingriff einen Rückfall. Ein weiterer Verdacht auf Leishmaniose bei einem Pferd aus Gießen wurde im Jahr 2006 dokumentiert. Auch in einem Rind und weiteren Pferden aus der Schweiz und Südwestdeutschland wurden 2009 autochthone, kutane Leishmaniosen beschrieben. Die Erreger dieser Fälle konnten jedoch inzwischen als $L$. siamensis identifziert werden [Müller et al. 2009, Lobsiger et al. 2010]. Weitere solcher equinen Fälle wurden kürzlich auch in den USA berichtet [Reuss et al. 2012]. Leishmania siamensis ist eine neu beschriebene Art, die 2008 in Thailand entdeckt wurde und dort
VL bei Menschen verursacht [Sukmee et al. 2008]. In den folgenden Jahren wurden etliche weitere humane Fälle, u.a. auch als HIV+ Koinfektion, in Thailand bekannt [Kongkaew et al. 2007, Maharom et al. 2008, Suankratay et al. 2010, Chusri et al. 2012, Bualert et al. 2012, Leelayoova et al. 2013]. Desweiteren liegen weitere bisher ungeklärte Fälle, die verursachende Leishmanienart betreffend, in Martinique von 1992 (humane VL), Ghana von 2006 (hu- 
mane VL) und in Australien von 2004 (bei Kängurus) vor [Noyes et al. 2002, Villinski et al., 2008, Rose et al., 2004, Dougall et al. 2009].

Europa wurde erst kürzlich als „hot spot" für neu und wieder auftretende Infektionskrankheiten (,(re)-emerging infectious diseases" - EID) aufgrund von Klimaveränderung und Globalisierung (Reisen, Migration, weltweiter Handel) bezeichnet und es wurde die Notwendigkeit unterstrichen, die Monitoringsysteme entsprechend anzupassen. Die viszerale Leishmaniose wurde 2012 als EID in Europa deklariert und große gesundheitspolitische Auswirkungen werden prognostiziert [Lindgren et al. 2012]. Insbesondere Mittel- und Osteuropa werden in diesem Zusammenhang als besonders wichtig eingeschätzt und es wird in Betracht gezogen, die Leishmaniose als meldepflichtige Krankheit bei der
ECDC (European Centre for Disease Prevention and Control) einzuführen.

Für die Planung präventiver Maßnahmen und aufgrund der vielen noch nicht genau untersuchten Fälle der neuen Art $L$. siamensis erscheint es umso wichtiger, diese Art genauer zu erforschen sowie alle bekannten und neu auftretenden autochthonen Fälle in Deutschland (bzw. in Mitteleuropa) genau zu identifizieren und zu typisieren.

Ziel dieser Arbeit war es zum einen, einen erneuten Versuch zur Identifizierung der Pferdeprobe aus dem Jahr 2004 zu unternehmen. Zum anderen sollte die phylogenetische Einordnung dieser Probe sowie von $L$. siamensis in die bestehenden Arten vorgenommen werden. Dazu wurden mehrere DNAMarker untersucht, für die in der Literatur und bei der Sequenzdatenbank GenBank für die bekannten Leishma- niaarten schon Sequenzinformationen vorliegen.

\section{MATERIAL UND METHODEN}

Zu den für die Identifizierung und für phylogenetische Untersuchungen der Leishmanien am häufigsten verwendeten genetischen Markern gehören die sog. Internal Transcribed Spacer (ITS1, ITS2) sowie die kleine Untereinheit (18S) der ribosomalen DNA, die DNA Polymerase $\alpha$, die große Untereinheit der RNA Polymerase II, das Cytochrom b Gen (cytb) und das Heatshock Protein 70 Gen (hsp70). Zu diesen Markern liegen auch die meisten Einträge bei der Sequenzdatenbank GenBank des National Center for Biotechnology Information (NCBI) vor. Diese bereits veröffentlichten Sequenzen von Leishmanien und anderen Vertretern der Ordnung Kinetoplastidae sowie die Informationen zu den verschiedenen

\begin{tabular}{|c|c|c|c|c|c|}
\hline Marker & Name & Sequenz 5'-3' & $\mathbf{T}_{\text {an }}$ & Größe & Referenz \\
\hline ITS1 rDNA & $\begin{array}{l}\text { LITSR } \\
5.8 S\end{array}$ & $\begin{array}{l}\text { CTGGATCATTTTCCGATG } \\
\text { TGATACCACTTATCGCACTT }\end{array}$ & $53^{\circ} \mathrm{C}$ & ca. 320 bp & El Tai et al. 2001 \\
\hline ITS2 rDNA & $\begin{array}{l}\text { 5.8SR } \\
\text { LITSV }\end{array}$ & $\begin{array}{l}\text { AAGTGCGATAAGTGGTA } \\
\text { ACACTCAGGTCTGTAAAC }\end{array}$ & $53^{\circ} \mathrm{C}$ & ca. 600 bp & El Tai et al. 2001 \\
\hline $\begin{array}{l}\text { SSU rDNA, } 1 . \\
\text { Runde }\end{array}$ & $\begin{array}{l}\text { R221 } \\
\text { R332 }\end{array}$ & $\begin{array}{l}\text { GGTTCCTTTCCTGATTTACG } \\
\text { GGCCGGTAAAGGCCGAATAG }\end{array}$ & $53^{\circ} \mathrm{C}$ & ca. $600 \mathrm{bp}$ & Schönian et al. 2003 \\
\hline $\begin{array}{l}\text { SSU rDNA, } 2 . \\
\text { Runde }\end{array}$ & $\begin{array}{l}\text { R223 } \\
\text { R333 }\end{array}$ & $\begin{array}{l}\text { TCCCATCGCAACCTCGGTT } \\
\text { AAAGCGGGCGCGGTGCTG }\end{array}$ & $65^{\circ} \mathrm{C}$ & ca. 353 bp & Schönian et al. 2003 \\
\hline hsp70 & $\begin{array}{l}\text { hsp70for } \\
\text { hsp70rev }\end{array}$ & $\begin{array}{l}\text { GACGGTGCCTGCСТACTTCAA } \\
\text { CCGСССАTGСТСТGGTACATC }\end{array}$ & $61{ }^{\circ} \mathrm{C}$ & ca. 1300 bp & $\begin{array}{l}\text { Garcia et al. 2004, } \\
\text { Montalvo et al. } 2010\end{array}$ \\
\hline cytb kDNA & $\begin{aligned} \text { Lcyt-S } & =\text { LCBF1 } \\
\text { Lcyt-R } & =\text { LCBR2 }\end{aligned}$ & $\begin{array}{c}\text { GGTGTAGGTTTTAGTYTAGG } \\
\text { CTACAATAAACAAATCATAATATRCAATT }\end{array}$ & $55^{\circ} \mathrm{C}$ & ca. 865 bp & $\begin{array}{c}\text { Kato et al. 2007, } \\
\text { Luyo-Acero et al. } 2004\end{array}$ \\
\hline $\begin{array}{l}\text { DNA Polyme- } \\
\text { rase } \alpha \text { (POLA) }\end{array}$ & $\begin{array}{l}\text { DNAP } \\
\text { DPO2 }\end{array}$ & $\begin{array}{c}\text { AACGAGCGCGCRCTGCTYGACTGG } \\
\text { GCCGAGGCAGCCATACAT }\end{array}$ & $52{ }^{\circ} \mathrm{C}$ & ca. 900 bp & Noyes et al. 2002 \\
\hline $\begin{array}{l}\text { RNA Polymerase } \\
\text { large subunit } \\
\text { (RPOIILS) }\end{array}$ & $\begin{array}{l}\text { RPOF1=RPO1F } \\
\text { RPOR1 }\end{array}$ & $\begin{array}{l}\text { GACACAGCCGTCAAGAC } \\
\text { GCAGCCGCACAATGCGCT }\end{array}$ & $45^{\circ} \mathrm{C}$ & ca. 1300 bp & Croan et al. 1997 \\
\hline
\end{tabular}


Arten und Stämmen wurden für eine Leishmania-Datenbank zusammengestellt. Hinzugefügt werden sollten die Sequenzen der in dieser Arbeit untersuchten Proben: die des deutschen Pferdes von 2004 T8316/2004 (MEQU/2004/DE/T8316), des Rezidivs T8316Rez/2004, eines weiteren Pferdes aus Deutschland ( $\mathrm{H}-2009)$ und des Rindes (MBOV/CH/2009/C1) aus der Schweiz (Lobsiger et al. 2010, Müller et al. 2009), der thailändischen L. siamensis-Kultur Stamm MHOM/ TH/2010/PCM2 (Bualert et al. 2012), der nicht identifizierten Probe MHOM/ MQ/1992/MAR1 der VL-Fälle in Martinique (Noyes et al. 2002) sowie um einige andere seltene Leishmanienarten aus Südamerika, zu denen bisher keine Sequenzinformationen für bestimmte Marker publiziert wurden, so u.a. $L$. enrietti MCAV/BR/1945/LV90. Dafür wurden die verschiedenen Marker mittels PCR amplifiziert und mit einem $A B I$ 3130xI Genetic Analyzer sequenziert.

Der PCR-Ansatz mit $25 \mu \mathrm{l}$ Volumen setzte sich wie folgt zusammen: 1 fach DreamTaq Puffer, 0,2 mM je dNTP, je 25 pmol forward- und reverse-Primer, 1 U Dream Taq DNA Polymerase und 2,0 $\mu$ I DNA (aus Kulturen mit einer Konzentration von $10 \mathrm{ng} / \mu \mathrm{l}$ ). Die DNA aus biologischem Material wurde unverdünnt unter Zugabe von 0,65 $\mu$ l Dimethylsulfoxid und $2,5 \mu \mathrm{l} 25 \mathrm{mM} \mathrm{MgCl}$ eingesetzt. Negativ- und Hemmkontrollen wurden standardmäßig durchgeführt. Für die verschiedenen Marker wurden unterschiedliche Thermocyclerprogramme etabliert, die sich in der Annealingtemperatur, der Dauer der einzelnen Schritte und der Anzahl der Zyklen unterscheiden. Die Primersequenzen, die Annealingtemperaturen sowie die entsprechenden Referenzen sind in Tabelle 2 zusammengefasst. Die PCR-Produkte wurden mittels Elektrophorese in 1\%igen Agarosegelen überprüft. Die Sequenzierung wurde mit dem Genetic Analyzer 3130xI von Applied Biosystems durch die Firma Services in Molecular Biology (SMB) $\mathrm{GmbH}$ durchgeführt.

Mit Hilfe des Programms BioEdit Version 7.1.11 und der Applikation ClustalW wurden Alignments der erhaltenen und der zuvor aus GenBank herausgesuchten Sequenzen für je- den der untersuchten genetischen Marker erstellt. Basierend auf diesen Alignments wurden in dem Programm MEGA Version 5.2.1 die phylogenetischen Bäume konstruiert. Die dabei verwendeten Methoden beruhen auf den Prinzipien Maximum Parsimony (kladistische Analyse) und Neighbour Joining (distanzbasierte Methode). Zusätzlich wurden Bootstrap-Analysen durchgeführt, um die Stabilität der Gruppierungen zu prüfen.

\section{ERGEBNISSE}

In der vorliegenden Arbeit wurden mehrere genetische Marker für verschiedene Leishmanienproben, von denen vermutet wird, dass es sich um $L$. siamensis handelt, mittels PCR amplifiziert und sequenziert. Dazu wurde die aus biologischem Material (Biopsien) autochthoner Leishmaniosefälle bei Pferden und einem Rind in Deutschland und der Schweiz gewonnene DNA verwendet, ebenso die DNA eines der thailändischen L. siamensis Referenzstämme sowie von weiteren $L$. siamensis-ähnlichen
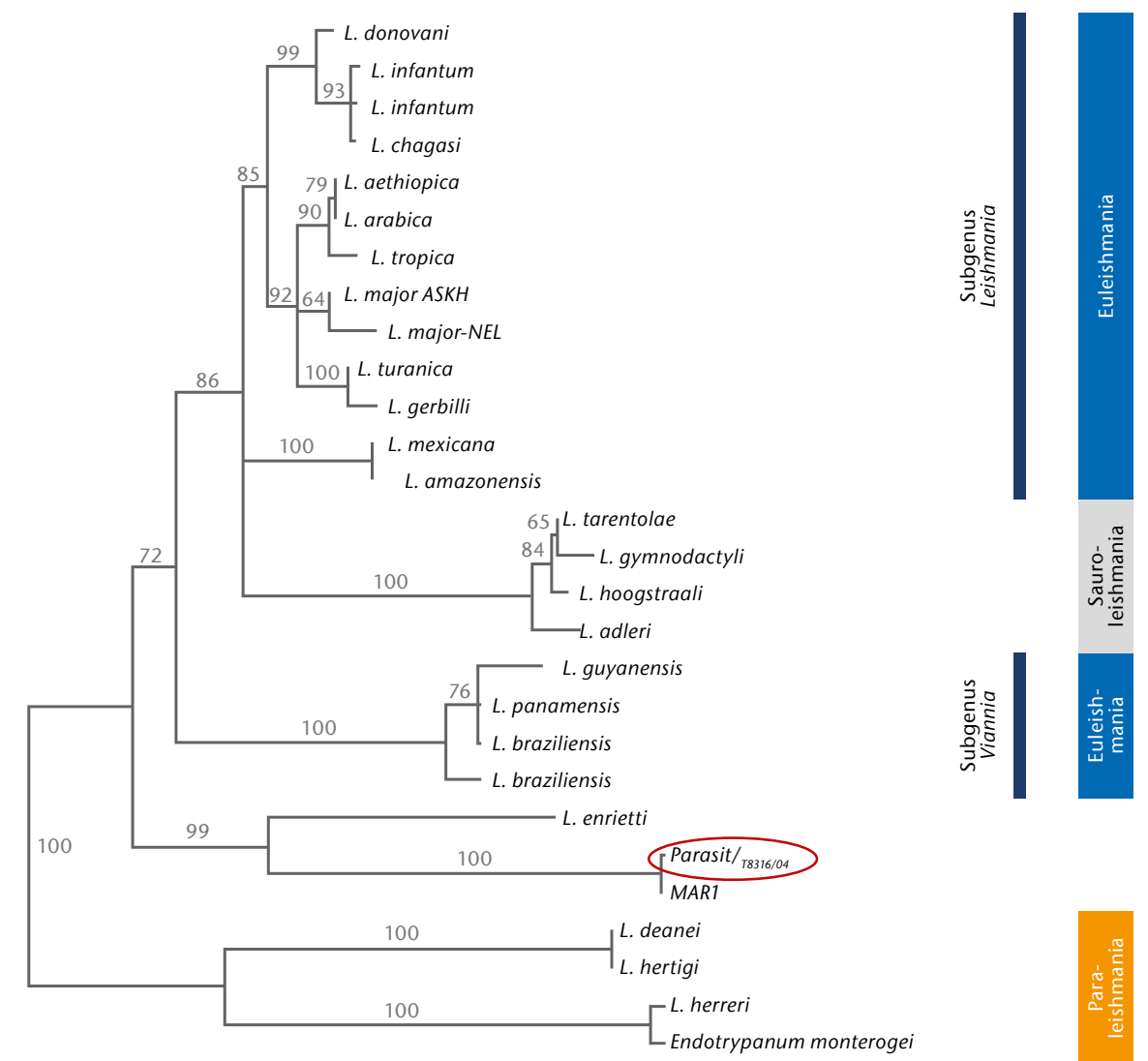

Abb. 3) Phylogenetische Position der Pferdeprobe T8316/2004 innerhalb der Gattung Leishmania. Der Stammbaum basiert auf den Sequenzen des DNA-Polymerase- $\alpha$-Gens und wurde mit der Parsimonieanalyse ermittelt. Bootstrapwerte (in Prozent) sind angegeben.
Stämmen, wie u.a. L. spec. aus humanen VL-Fällen auf Martinique und $L$. enrietti.

Mit der Software BioEdit wurden die Amplikon-Sequenzen und die Sequenzen sämtlicher Leishmanienarten und einiger Vertreter anderer Trypanosomatiden (Endotrypanum schaudinii, Endotrypanum monterogeii, Trypanosoma cruzi, Trypanosoma brucei, Crithidia fasciculata, Leptomonas costaricensis), sofern bei GenBank vorhanden, für jeden der untersuchten Marker mittels multiplen Alignments verglichen. Diese Alignments stellten die Basis für die Konstruktion der phylogenetischen Bäume dar. Um die Verzweigung und Einordnung der untersuchten DNA zweifach zu bestätigen, wurden die beiden Konstruktionsmethoden Maximum Parsimony und Neighbour Joining mit zusätzlicher Bootstrap-Analyse angewendet und somit zu jedem Marker zwei Bäume erstellt.

Eines der Hauptziele der Arbeit war die Identifizierung der Pferdeprobe aus dem Jahr 2004, die zum damaligen 


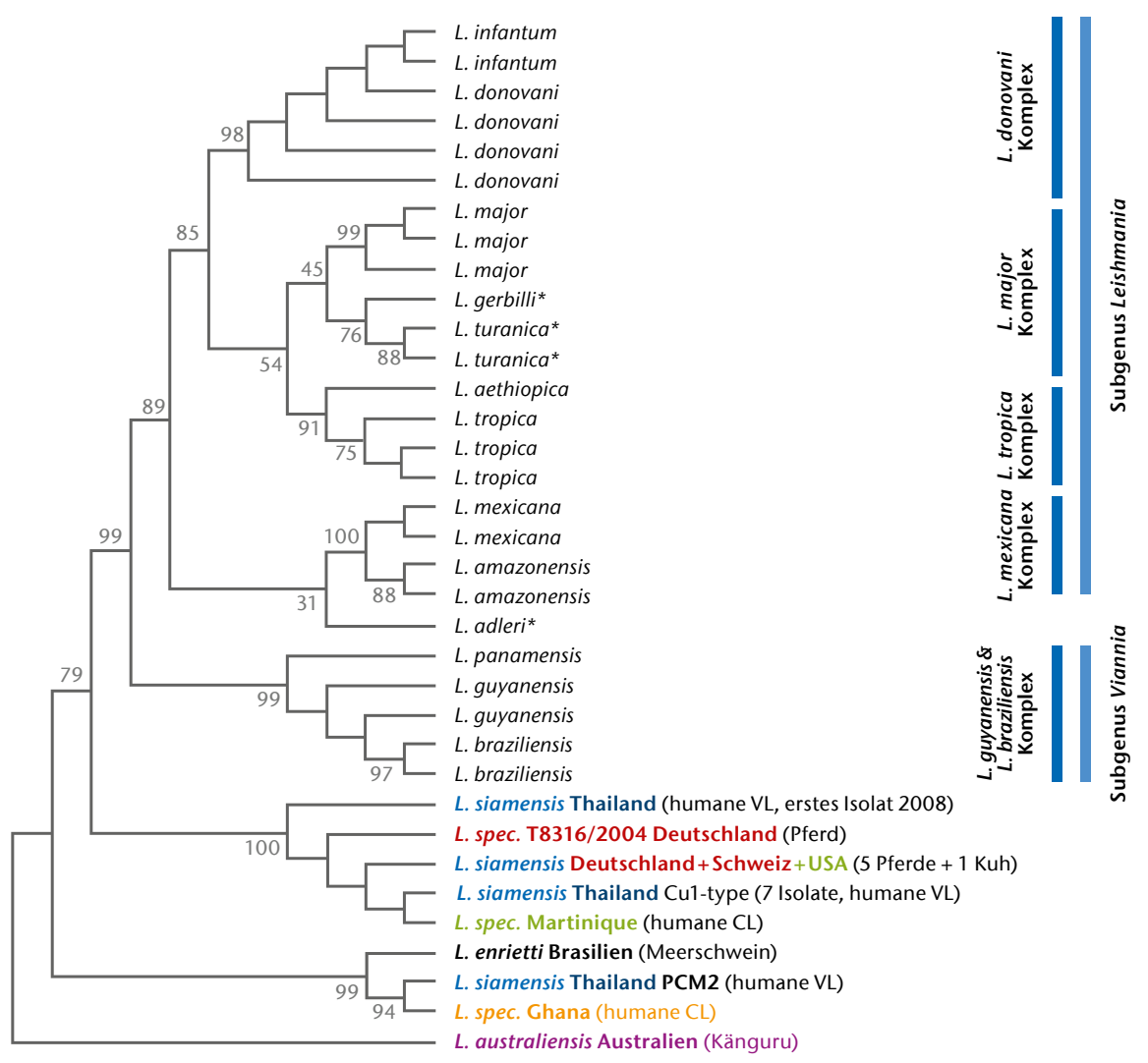

Abb. 4) Phylogenetische Position der Pferdeprobe T8316/2004 innerhalb der Gattung Leishmania. Der Stammbaum basiert auf den Sequenzen der ITS1-Region der ribosomalen DNA und wurde mit der distanzbasierten Neighbor-Joining Methode ermittelt. Bootstrapwerte (in Prozent) sowie die taxonomische Einordnung der einzelnen Arten in die zwei Untergattungen sowie die Artkomplexe sind angegeben. * nicht humanpathogene Arten

Zeitpunkt zwar der Gattung Leishmania, jedoch keiner bekannten Leishmaniaart zugeordnet werden konnte. Die Amplifikation der ITS1- Region zeigte zunächst, dass es sich bei dem unbekannten Parasiten um einen Vertreter derTrypanosomatiden handelt. Erst die Sequenzierung des DNA-Polymerase$\alpha$-Gens (POLA) ergab die Zugehörigkeit zur Gattung Leishmania. Es zeigte sich, dass dieser Parasit innerhalb des Stammbaums dieser Gattung eine basale Position einnimmt, d.h. relativ entfernt verwandt zu den anderen in Europa vorkommenden Leishmanien ist und eine eigenständige phylogenetische Gruppe mit L. enrietti, einem bei Meerschweinen in Südamerika auftretenden Parasiten, sowie dem 1992 in Martinique isolierten Stamm MAR1 eines humanen CL-Falls bildet (Abb. 3). Interessanterweise unterschieden sich die Sequenzen der Pferdeprobe und des MAR1-Stammes nur durch einen einzigen Basenaustausch.

Erst die erneute Sequenzanalyse der ITS1-Region in diesem Jahr ergab, dass es sich bei der Pferdeprobe um die im Jahr 2008 neu beschriebene Art L. siamensis handelt. Es zeigte sich, dass die sehr variable ITS1-Region bei folgenden Proben identisch war: der Pferdeprobe T8316/2004 aus Deutschland, den 2009 in der Schweiz und Deutschland aufgetretenen equinen und bovinen Leishmaniosefällen [Müller et al. 2009, Lobsiger et al. 2010], dem erst kürzlich in den USA beschriebenen Fällen von Pferdeleishmaniose [Reuss et al. 2012], dem bisher nicht identifizierten Leishmania-ähnlichen VL-Stamm aus Martinique [Noyes et al. 2002] sowie 7 der 8 in Thailand beschriebenen humanen VL-Fälle aus den Jahren 2008-2012. Interessanterweise scheint es zwei phylogenetische Gruppen innerhalb von $L$. siamensis zu geben, da sich einer der VL-Stämme aus Thailand in der ITS1-Sequenz deutlich von der ersten Gruppe unterscheidet und eine nahezu identische Sequenz zu einem Leishmania-ähnlichen Isolat, das bei humanen CL-Fällen in Ghana gefunden wurde und bisher auch nicht zugeordnet werden konnte, aufweist.
Die größte Ähnlichkeit dieser zweiten Gruppe bestand zu L. enrietti. Wie in dem Stammbaum in Abb. 4 zu sehen ist, sind die zwei L. siamensis Gruppen nur entfernt verwandt zu den anderen humanpathogenen Leishmaniaarten der Untergattungen Viannia und Leishmania. Innerhalb der Gattung Leishmania sind die auch erst 2004 bei Kängurus gefundenen Parasiten (L. australiensis) phylogenetisch am weitesten entfernt. In dem mit der Parsimonie-Analyse erstellten Baum (hier nicht gezeigt) ist L. australiensis Teil der zweiten Gruppe, so wie auch L. enrietti.

Die meisten publizierten Sequenzen liegen z.Z. für die ITS1-Region vor, einschließlich der L. siamensis Stämme. Der SSU-Marker ist derjenige mit dem geringsten Differenzierungspotential, gibt aber wichtige Aufschlüsse, wenn es um die Einordnung der Isolate auf Gattungsebene geht - auch für diesen Marker liegen viele Sequenzen vor, problematisch ist jedoch, dass z.T. unterschiedliche Teilfragmente durch die einzelnen Autoren sequenziert wurden und die Sequenzen nicht homolog sind. Die Marker cytb und hsp70 eignen sich wiederum sehr gut für die Differenzierung auf der Artebene. Grundsätzlich zeigten sowohl ITS1 als auch hsp70 und cytb die Ähnlichkeit von $L$. siamensis zu $L$. enrietti und L. spec. aus Martinique (MAR1). Für L. australiensis und den L. siamensisähnlichen Stamm aus Ghana liegen leider bisher keine Sequenzen für cytb und hsp70 vor. Alle Marker ordneten $L$. siamensis (mit Ausnahme des Stammes PCM2) als monophyletische Gruppe innerhalb der Gattung Leishmania ein, die möglicherweise eine dritte bisher nicht klassifizierte Untergattung neben den Subgenera Viannia und Leishmania darstellt.

\section{DISKUSSION}

Zur Untersuchung autochthoner Leishmaniosefälle bei Nutztieren in Deutschland und der Schweiz in Zusammenhang mit der neuen Leishmanienart $L$. siamensis wurden mittels PCR sieben verschiedene genetische Marker mehrerer entsprechender Proben amplifiziert und sequenziert sowie mit den Sequenzen anderer 


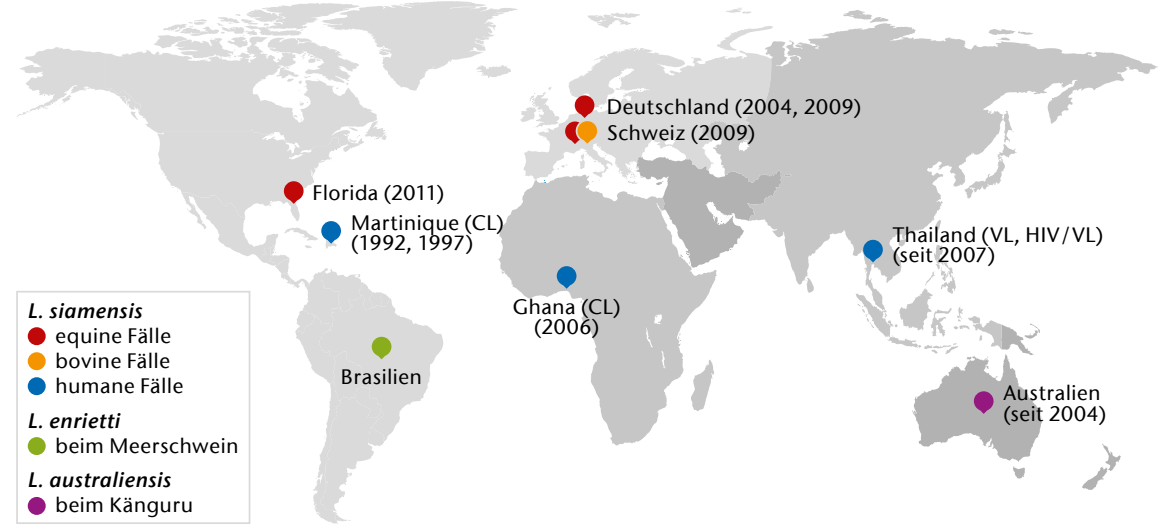

Abb. 5) Bisherige Leishmaniose-Fälle, die durch L. siamensis bzw. die nah verwandten Arten L. enrietti und L. australiensis verursacht wurden. In Klammern ist das Jahr des Auftretens der Erkrankung angegeben. $C L$ - kutane Leishmaniose, $V L$ - viszerale Leishmaniose.

Leishmaniaarten verglichen. So konnte u.a. der Erreger der schon 2004 diagnostizierten Hautleishmaniose bei einem Pferd aus Deutschland als L. siamensis identifiziert werden. Des Weiteren konnten wir zeigen, dass es sich bei den 1992 in Martinique aufgetretenen VL-Fällen bei Menschen sowie den 2006/2007 in Ghana diagnostizierten humanen CL-Fällen auch um L. siamensis handelt, d.h. dass diese Art schon viel länger als Erreger der Leishmaniose zirkuliert. Leishmania siamensis ist somit die einzige Leishmaniaart, die auf vier bzw. fünf Kontinenten (Europa, Asien, Süd- und Nordamerika, Afrika) vorkommt, d.h. die weiteste Verbreitung aller Arten aufweist und trotzdem erst vor kurzem als Auslöser von Leishmaniosen beschrieben wurde (Abb. 5). Interessanterweise ist auch das Spektrum der klinischen Bilder sehr komplex einerseits sind sowohl Menschen als auch Tiere betroffen, andererseits treten sowohl kutane als auch viszerale Fälle auf, desweiteren auch Koinfektionen mit HIV. In Mitteleuropa gab es allerdings bisher nur Fälle bei Nutztieren, das Auftreten von humanen Infektionen kann aber auch hier nicht ausgeschlossen werden, so dass es sich um eine neu auftretende zoonotische Infektionskrankheit handeln könnte. Völlig unklar ist bis jetzt, wie L. siamensis übertragen wird, d.h. welche Vektoren dafür in Frage kommen, das betrifft nicht nur die Fälle in Mitteleuropa. Da in Deutschland und der Schweiz bisher nur Ph. mascittii und Ph. perniciosus auftreten, könnte man vermuten, dass eine oder beide dieser Sandmückenarten als Vektor fungieren, allerdings gibt es noch keinerlei Untersuchungen zum Transmissionspotential dieser Sandmückenarten für L. siamensis. Des Weiteren gibt es bisher auch noch keinerlei Erkenntnisse dazu, ob Ph. mascittii überhaupt Leishmanien überträgt.

Die Ergebnisse der vorliegenden Arbeit zeigten anhand der Marker cytb, hsp70 und ITS1 auch, dass es zwei unterschiedliche phylogenetische Gruppen innerhalb von $L$. siamensis gibt, was auch kürzlich in einer Studie der thailändischen Fälle festgestellt wurde [Leelayoova et al. 2013]. Interessanterweise scheint einer der L. siamensis Genotypen aus Thailand (PCM2) enger mit $L$. enrietti und dem bisher nicht identifizierten Parasiten in Ghana verwandt zu sein als mit allen anderen $L$. siamensis Stämmen. Eine enge Verwandtschaft des PCM2 Stammes zu L. enrietti zeigte sich auch bei der Sequenzanalyse von drei anderen proteinkodierenden Genen [Bualert et al. 2012]. Die Sequenzvariabilität innerhalb der ITS1-Region zwischen diesen beiden Gruppen entspricht den Werten, die sonst zwischen verschiedenen Leishmaniaarten zu finden sind, so dass es nicht auszuschließen ist, dass es sich hier um Subspezies, wenn nicht sogar verschiedene Arten, handelt. Auch die phylogenetisch-taxonomische Position der bei Kängurus auftretenden Leishmanien ist bislang noch nicht geklärt. Unsere Ergebnisse weisen darauf hin, dass diese Parasiten eng mit der zweiten Gruppe von L. siamensis (einschließlich $L$. enrietti) verwandt sind, so dass auch hier sich die Frage stellt, ob es sich tatsächlich bei dieser Gruppe um drei verschiedene Arten - L. enrietti, L. siamensis und L. australiensis - handelt. In diesem Fall wäre $L$. siamensis paraphyletisch.

Um diese Fragen klären zu können, müssen einerseits viel mehr Stämme dieser drei Arten untersucht werden, um die Sequenzvariabilität innerhalb der einzelnen Arten abschätzen zu können. Problematisch ist des Weiteren, dass nicht für alle Arten und auch die untersuchten $L$. siamensis Stämme sowie einige der bisher nicht identifizierten L. siamensis-ähnlichen Stämme die Sequenzen für alle untersuchten Marker vorliegen, so dass nicht mit einem identischen Stammset für alle Marker gearbeitet werden konnte. Diese fehlenden Arten müssen in zukünftigen Studien für die entsprechenden Marker komplettiert werden. Bedauerlicherweise existiert von vielen der als $L$. siamensis identifizierten Proben kein Material (Biopsie, DNA oder Kultur) mehr.

Phylogenetische Studien sind wichtig, um bestimmte biologische Eigenschaften der einzelnen Taxa vergleichen zu können und auch Rückschlüsse zum Ursprung und zur Verbreitung der Organismen zu ziehen. Die Möglichkeit, dass sich Deutschland und andere Länder in Mitteleuropa als neue Foci für Leishmaniosen entwickeln könnten, ist in jedem Fall ernst zu nehmen. Deshalb ist es wichtig, weitere Forschung zu diesem Thema zu betreiben. Die Studien zu L. siamensis als Verursacher von autochthonen Leishmaniosen sind ein erster Schritt in diese Richtung.

Um die Gefährdung von Menschen in Mitteleuropa besser einschätzen zu können, sind weitere Untersuchungen zur Virulenz, Wirtspräferenz, Medikamentensensitivität und zum Transmissionszyklus von $L$. siamensis (insbesondere der europäischen Parasiten) notwendig. Diese Studien erfordern das erfolgreiche Ansetzen einer Kultur, was bis jetzt leider noch für keinen der in Deutschland und der Schweiz aufgetretenen Fälle gelungen ist. Epidemiologische Studien einschließlich der Surveillance, dem Screening von Nutztieren sowie der Menschen in den be- 
troffenen Regionen, der genetischen Typisierung und Untersuchungen zum Ursprung und der Verbreitung der Infektionen und die Identifizierung des Vektors sind die Hauptaufgaben für eine effektive Prävention und Kontrolle dieser neuen Infektionskrankheit in Mitteleuropa.

\section{DANKSAGUNG}

Wir möchten uns bei Harry Noyes (Institute of Integrativ Biology, Dept. of Functional and Comparative Genomics, University of Liverpool, UK), Norbert Müller (Institut für Parasitologie der Vetsuisse Fakultät und der Medizinischen Fakultät, Universität Bern), Mohamed Kasbari (ANSES - Agence Nationale de Sécurité Sanitaire, Laboratoire de Santé Animale, Mission Leishmanioses \& Phlébotomes, Maisons Alfort, France), Elisa Cupolillo (Instituto Oswaldo Cruz - Fiocruz, Laboratório de Pesquisa em Leishmaniose, Rio de Janeiro, Brasilien) und Christophe Ravel (Université de Montpellier 1, Faculté de Médecine, France) für die Zurverfügungstellung von DNA verschiedener Leishmania-Proben bedanken.

\section{LITERATUR}

Beyreder J. (1962): Ein Fall von Leishmaniose in Niederösterreich. Wien Med Wochenschr 115:900-901.

Bogdan C, Schönian G, Banuls AL, Hide M, Pratlong F, Lorenz E, Röllinghoff M, Mertens R. (2001): Visceral Leishmaniasis in a german child who had never entered a known endemic area: case report and review of the literature. Clin Inf Dis 31: 302-306.

Bualert L, Charungkiattikul W, Thongsuksai P, Mungthin M, Siripattanapipong S, Khositnithikul R, Naaglor T, Ravel C, El Baidouri F, Leelayoova S. (2012): Case report: Autochthonous dissiminated dermal and visceral leishmaniasis in an AIDS patient, Southern Thailand, caused by Leishmania siamensis. Am J Trop Med Hyg 86(5): 821-824.

Callot J. (1959): Présence de Phlebotomus larroussei en Alsace. Annales de Parasitologie Humaine et Comparée 25:112.

Chusri S, Hortiwakul T, Silpapojakul K, Sirivasatien P. (2012): Case report: Consecutive cutaneous and visceral leishmaniasis manifestations involving a novel Leishmania species in two HIV patients in Thailand. Am J Trop Med Hyg 87(1): 76-80

Croan DG, Morrison DA, Ellis JT (1997): Evolution of the genus Leishmania revealed by comparison of DNA and RNA polymerase gene sequences. Mol Biochem Parasitol. Nov;89(2):149-59.

Depaquit J, Naucke TJ, Schmitt $\mathrm{C}$, Ferté $\mathrm{H}$, Léger $\mathrm{N}$. (2005): A molecular analysis of the subgenus Transphlebotomus Artemiev, 1984 (Phlebotomus, Diptera, Psychodidae) inferred from ND4 mtDNA with new northern records of Phlebotomus mascittii Grassi, 1908. Parasitol Res 43: 113-116.
Diaz-Espineira MM, Slappendel RJ. (1997): A case of autochthonous canine leishmaniasis in The Netherlands. Vet Q 19: 69-71.

Dornbusch HJ, Urban C, Kerbl C, Lackner H, Schwinger W, Sovinz P, Zottner H, Aspöck H. (1999): Viszerale Leishmaniose bei einem 10 Monate alten österreichischen Mädchen. XXXIII. Tagung der Österreichischen Gesellschaft für Tropenmedizin und Parasitologie (1999).

Dougall A, Shilton C, Low Choy J, Alexander B, Walton S. (2009): New reports of Australian cutaneous leishmaniasis in northern Australian macropods. Epidemiol Infect 137:1516-1520.

El Tai NO, El Fari M, Mauricio I, Miles MA, Oskam L, El Safi SH, Presber WH, Schönian G.(2001): Leishmania donovani: intraspecific polymorphisms of Sudanese isolates revealed by PCR-based analyses and DNA sequencing. Exp Parasitol. Jan 97(1):35-44.

Fischer D, Moeller P, Thomas SM, Naucke TJ, Beierkuhnlein C. (2011): Combining climatic projections and dispersal ability: a method for estimating the responses of sandfly vector species to climate change. PLoS NTD 5(11): e1407.

Fischer D, Thomas SM, Beierkuhnlein C. (2010): Temperature-derived potential for the establishment of phlebotomine sandflies and visceral leishmaniasis in Germany. Geospatial Health 5(1): 59-69.

Galli-Valerio B. (1912): Beobachtungen über Culiciden und Mitteilung über das Vorkommen von Phlebotomus papatasi (Scop.) im Kanton Waadt. Zentralblatt für Bakteriologie 43: 222-226.

Garcia L, Kindt A, Bermudez H, Llanos-Cuentas A, De Doncker S, Arevalo J, Wilber Quispe Tintaya K, Dujardin JC. (2004): Culture-independent species typing of neotropical Leishmania for clinical validation of a PCRbased assay targeting heat shock protein 70 genes. I Clin Microbiol. May;42(5):2294-7.

Gothe R. (1991): Leishmaniosen des Hundes in Deutschland: Erregerfauna und -biologie, Epidemiologie, Klinik, Pathogenese, Diagnose, Therapie und Prophylaxe. Kleintierpraxis 36: 69-84.

Grimm F, Knechtli R, Gessler M, Jenni L. (1990): Biology of sandflies in southern Switzerland. Rev Suisse Zool 97: 778-779.

Grimm F, Gessler M, Jenni L. (1993): Aspects of sandfly biology in Southern Switzerland. Med Vet Entomol 7: $170-176$

Harms G, Schönian G, Feldmeier H. (2003): Leishmaniasis in Germany. Emerg Inf Dis 9 (7): 872-875.

Kato $\mathrm{H}$, Uezato H, Gomez EA, Terayama Y, Calvopiña M, Iwata H, Hashiguchi Y. (2007): Establishment of a mass screening method of sand fly vectors for Leishmania infection by molecular biological methods. Am J Trop Med Hyg. Aug 77(2):324-9.

Knechtli R \& Jenni L. (1990): Experimental transmission of Leishmania infantum by the bite of Phlebotomus perniciosus from Switzerland. Acta Tropica 47:213-216.

Koehler K, Stechele M, Hetzel U, Domingo M, Schönian G, Zahner H, Burkhardt E. (2002): Cutaneous leishmaniosis in a horse in southern Germany caused by Leishmania infantum. Vet Parasitol 109: 9-17.

Kollaritsch H, Emminger W, Zaunschirm A, Aspöck H. (1989): Suspected autochthonous kala-azar in Austria. Lancet 22: 901-902.

Kongkaew W, Siriarayaporn P, Leelayoova S, Supparatpinyo K, Areechokchai D, Duang-ngern $P$, Chanachai K, Sukmee T, Samung Y, Sridurongkathum P. (2007): Autochthonous visceral leishmaniasis: a report of a second case in Thailand. Southeast Asian J Trop Med Publ Health 38(1): 8-12.
Kuhls K, Sinning D, Rauhut F, Köhler K, Litzke LF, Schönian G. (2013): Leishmania siamensis as the cause of autochthonous cutaneous leishmaniasis of horses in Germany - a new emerging zoonotic disease? WorldLeish 5 2013. Abstract

Leelayoova S, Siripattanapipong S, Hitakarun A, Kato $\mathrm{H}$, Tan-ariya P, Siriyasatien P, Osatakul S, Mungthin M. (2013): Multilocus characterization and phylogenetic analysis of Leishmania siamensis isolated from autochthonous visceral leishmaniasis cases, southern Thailand. BMC Microbiology 13:60

Lindgren E. Andersson Y, Suk JE, Sudre B, Semenza JC. (2012): Monitoring EU emerging infectious disease risk due to climate change. Science 336:418-419.

Litzke, L-F, Köhler, K, Schoenian, G, Zahner, H. (2006): Autochthone Hautleishmaniose beim Pferd. Tagung "Diagnostik, Epidemiologie und Bekämpfung von Parasitosen “ der Fachgruppe Parasitologie und parasitäre Krankheiten der Deutschen Veterinärmedizinischen Gesellschaft, Wetzlar, 2006.

Lobsiger L, Müller N, Schweizer T, Frey CF, Wiederkeh D, Zumkehr B, Gottstein B. (2010): An autochthonous case of cutaneous bovine leishmaniasis in Switzerland. Vet Parasitol 169: 408-414.

Lollaritsch H, Emminger W, Zanschirm A, Aspöck H. (1989): Suspected autochthonous kala-azar in Austria [letter]. Lancet 1: 901-902.

Luyo-Acero GE, Uezato H, Oshiro M, Takei K, Kariya K, Katakura K, Gomez-Landires E, Hashiguchi Y, Nonaka S (2004): Sequence variation of the cytochrome b gene of various human infecting members of the genus Leishmania and their phylogeny. Parasitology May128(Pt 5):483-91.

Maharom P, Siripattanapipong S, Mungthin M, Naaglor T, Sukkawee R, Pudkorn R, Wattana W, Wanachiwanawin D, Areechokchai D, Leelayoova S. (2008): Visceral leishmaniasis caused by Leishmania infantum in Thailand. Southeast Asian J Trop Med Public Health 39(6): 88-990.

Maroli M, Rossi L, Baldelli R, Capelli G, Ferroglio E, Genchi C, Gramiccia M, Mortarino M, Pietrobelli M, Gradoni L. (2008): The northward spread of leishmaniasis in Italy: evidence from retrospective and ongoing studies on the canine reservoir and phlebotomine vectors. Trop Med Int Health 13(2): 256-264.

Mazzi R. (1976): Kutane Leishmaniose: Autochthoner Fall in der Schweiz? Dermatologica 153: 104-105.

Menn B, Lorentz S, Naucke TJ. (2010): Imported and travelling dogs as carriers of canine vector-borne pathogens in Germany. Parasites \& Vectors 3, 34

Montalvo AM, Fraga I, Monzote L, Montano I, De Doncker S, Dujardin JC, Van der Auwera G. (2010): Heat-shock protein 70 PCR-RFLP: a universal simple tool for Leishmania species discrimination in the New and Old World. Parasitology Jul;137(8):1159-68.

Müller N, Welle M, Lobsiger L, Stoffel MH, Kühni Boghenbor K, Hilbe M, Gottstein B, Frey CF, Geyer C, von Bomhard W. (2009): Occurence of Leishmania sp. in cutaneous lesions of horses in Central Europe. Vet Parasitol 166: 346-351.

Naucke TJ, Pesson B. (2000): Presence of Phlebotomus (Transphlebotomus) mascittii Grassi, 1908 (Diptera: Psychodidae) in Germany. Parasitol Res 86: 335-336.

Naucke TJ. (2002): Leishmaniose, eine Tropenkrankheit und deren Vektoren (Diptera, Psychodidae, Phlebotominae) in Mitteleuropa. Denisia 6 Nr. 184: 163-178.

Naucke TJ, Menn B, Massberg D, Lorentz S. (2008): Sandflies and leishmaniasis in Germany. Parasitol Res (Suppl 1) 103: 65-68 
Naucke TJ, Lorentz S, Rauchenwald F, Aspöck H. (2011): Phlebotomus (Transphlebotomus) mascittii Grassii, 1908, in Carinthia: first record oft he occurrence of sandflies in Austria (Diptera: Psychodidae: Phlebotominae). Parasitol Res 109(4): 1161-1164.

Naucke TJ \& Schmitt C. (2004): Is leishmaniasis becoming endemic in Germany? Int J Microbiol 293 (Suppl. 37):179-181.

Noyes H, Pratlong F, Chance M, Ellis J, Lanotte G, Dedet IP. (2002): A previously unclassified trypanosomatid responsible for human cutaneous lesions in Martinique (French West Indies) is the most divergent member of the genus Leishmania ss. Parasitology 124: 17-24.

Reuss SM, Dunbar MD, Calderwood Mays MB, Owen IL, Mallicote MF, Archer LL, Wellehan Jr JFX. (2012): Autochthonous Leishmania siamensis in Horse, Florida, USA. Emerging Infectious Diseases 18(9): 1545-46.

Rose K, Curtis I, Baldwin T, Mathis A, Kumar B, Sakthianandeswaren A, Spurck T, Low Choy J, Handman E. (2004): Cutaneous leishmaniasis in red kangaroos isolation and characterization of the causative organisms. Int Parasitol 34: 655-664.

Schawalder P. (1977): Leishmaniose bei Hund und Katze. Autochthone Fälle in der Schweiz. Kleintierpraxis 22: $237-246$

Schönian G, Nasereddin A, Dinse N, Schweynoch C, Schallig HD, Presber W, Jaffe CL. (2003): PCR diagnosis and characterization of Leishmania in local and imported clinical samples. Diagn Microbiol Infect Dis Sep 47(1):349-58

Slappendel RJ. (1988): Canine leishmaniasis: a review based on 95 cases in The Netherlands. Vet Q 10: 1-16.

Suankratay C, Suwanpimolkul G, Wilde H, Siriyasatien P.(2010): Case report: Autochthonous visceral leishmaniasis in a human immunodeficiency virus (HIV) infected patient: the first in Thailand and review of the literature. Am J Trop Med Hyg 82(1): 4-8.

Sukmee T, Siripattanapipong S, Mungthin M, Worapong J, Rangsin R, Samung Y, Kongkaew W, Bumrungsana K, Chanachai K, Apiwathanasorn C, Rujirojindakul P, Wattanasri S, Ungchusak K, Leelayoova S. (2008): A suspected new species of Leishmania, the causative agent of visceral leishmaniasis in a Thai patient. Int J Parasitol 38: 617-622.

Villinski JT, Klena JD, Abbassy M, Hoel DF, Puplampu N, Mechta S, Boakye D, Raczniak G. (2008): Evidence for a new species of Leishmania associated with a focal disease outbreak in Ghana. Diagnos Microbiol Infec Dis 60: 323-327.

Vogel R. (1931): Beobachtungen über blutsaugende Zweiflügler im Kanton Tessin. Zool Anz): 1-3.

Weitzel T, Mühlberger N, Jelinek T, Schunk M, Ehrhardt $S$ et al. (2005): Imported leishmaniasis in Germany 2001-2004: data of the SIMPID surveillance network.

Eur I Clin Micribiol Infect Dis 24: 471-476

World Health Organization (2010): Control of the leishmaniases: report of a meeting of the WHO Expert Committee on the Control of Leishmaniases, Geneva, 22-26 March 2010, WHO technical support series (2010), No. 949.

Wiwanitkit V. (2011): Bone marrow leishmaniasis: review of situation in Thailand. Asian Pacific J Trop Med 757-759.

\section{AUTOREN}

Denise Sinning, B.Sc.

Biosystemtechnik/Bioinformatik

Labor für Molekulare Biotechnologie und

Funktionelle Genomik

Technische Hochschule Wildau [FH]

sinning@th-wildau.de

\section{Dr. Kernt Köhler}

Institut für Veterinär-Pathologie de

Justus-Liebig-Universität Gießen

Kernt.Koehler@vetmed.uni-giessen.de

Prof. Dr. Lutz-Ferdinand Litzke

Klinik für Pferde (Chirurgie) der

Justus-Liebig-Universität Gießen

lutz-f.litzke@vetmed.uni-giessen.de

\section{Dr. rer. nat. Gabriele Schönian}

Institut für Mikrobiologie und Hygiene,

Charité Universitätsmedizin Berlin

Gabriele.Schoenian@charite.de

Prof. Dr. sc. hum. Marcus Frohme

Labor für Molekulare Biotechnologie

und Funktionelle Genomik

Technische Hochschule Wildau [FH]

marcus.frohme@th-wildau.de

\section{Dr. rer. nat. Katrin Kuhls (korrespondierender Autor)}

Labor für Molekulare Biotechnologie und

Funktionelle Genomik

Technische Hochschule Wildau [FH]

Katrin.Kuhls@th-wildau.de 